\title{
Using Polar Coronal Hole Area Measurements to Determine the Solar Polar Magnetic Field Reversal in Solar Cycle 24
}

\author{
N. Karna • S.A. Hess Webber • W.D. Pesnell
}

Received: 3 December 2013 / Accepted: 22 April 2014 / Published online: 6 May 2014

(C) Springer Science+Business Media Dordrecht (outside the USA) 2014

\begin{abstract}
An analysis of solar polar coronal hole (PCH) areas since the launch of the Solar Dynamics Observatory (SDO) shows how the polar regions have evolved during Solar Cycle 24. We present PCH areas from mid-2010 through 2013 using data from the Atmospheric Imager Assembly (AIA) and Helioseismic and Magnetic Imager (HMI) instruments onboard SDO. Our analysis shows that both the northern and southern $\mathrm{PCH}$ areas have decreased significantly in size since 2010. Linear fits to the areas derived from the magnetic-field properties indicate that, although the northern hemisphere went through polar-field reversal and reached solar-maximum conditions in mid-2012, the southern hemisphere had not reached solar-maximum conditions in the polar regions by the end of 2013. Our results show that solar-maximum conditions in each hemisphere, as measured by the area of the polar coronal holes and polar magnetic field, will be offset in time.
\end{abstract}

Keywords Coronal holes $\cdot$ Solar cycle $\cdot$ Magnetic reversal $\cdot$ Observation

\section{Introduction}

Determining the timing of solar minimum and maximum has long been the domain of the sunspot number. However, sunspots are rarely seen above $40^{\circ}$ latitude in either hemisphere

The Many Scales of Solar Activity in Solar Cycle 24 as seen by SDO

Guest Editors: Aaron Birch, Mark Cheung, Andrew Jones, and W. Dean Pesnell

N. Karna · S.A. Hess Webber · W.D. Pesnell $(\bowtie)$

NASA Goddard Space Flight Center, Greenbelt, MD, USA

e-mail: william.d.pesnell@ nasa.gov

N. Karna $\cdot$ S.A. Hess Webber

George Mason University, Fairfax, VA, USA

N. Karna

e-mail: nkarna@masonlive.gmu.edu

S.A. Hess Webber

e-mail: shessweb@masonlive.gmu.edu 
(Sawtell Cameron, 1957). The solar dynamo has a multiplicity of properties, each following the trend of the sunspot number, but with departures in phasing that provide clues about how the dynamo operates. Features at high latitudes sense a different aspect of the solar magnetic field. Models of the solar dynamo can use the variations of these features to better reproduce the observed magnetic field.

Solar features that vary out of phase with the sunspot number are also interesting. By reaching a maximum when sunspots are absent, they act as a probe during those featureless times. By going through zero near solar maximum, they provide another way to set the timing of that extremum. Two candidates for such features are the solar polar magnetic field and the area of the polar coronal holes (PCHs). The polar magnetic field has been measured since 1975 at the Wilcox Solar Observatory (WSO: Scherrer et al., 1977) and these measurements have been combined with models to study the evolution of the solar magnetic field (DeRosa, Brun, and Hoeksema, 2012).

Coronal holes are dark regions where the corona has low emission in extreme ultraviolet and soft-X-ray wavelengths, allowing a view to almost the surface of the Sun (Bohlin, 1977; Bilenko, 2002; Cranmer, 2009). Polar coronal holes are long-lived coronal holes surrounding the rotational poles of the Sun. The PCH areas show a temporal behavior similar to that of the polar magnetic field. During solar maximum, as the polar field decreases through zero and reverses its polarity, the PCHs shrink. As the cycle progresses back to solar minimum, the polar field reaches maximum strength (Babcock, 1959), and the PCHs reemerge and expand (Waldmeier, 1981; Webb, Davis, and McIntosh, 1984; Kirk et al., 2009). Once the polar holes are well established, the remaining equatorial holes in each hemisphere tend to have the same polarity as the polar hole in that hemisphere (Bohlin and Sheeley, 1978).

Hess Webber et al. (2014), hereafter Article I, showed that the PCH area and magneticfield strength vary in concert during Solar Cycle 23, but they are not well correlated in magnitude. In fact, the PCH areas have the same size between the two solar minima that were considered, while the polar-field strength decreased by half. However, the Extreme ultraviolet Imaging Telescope (EIT) data used in Article I had significant systematic fluctuations that are similar in amplitude to the solar-cycle variation. For this study we use data from the SDO Atmospheric Imager Assembly (AIA) and Helioseismic and Magnetic Imager (HMI), which have a smaller annual modulation than the EIT data, to investigate the simultaneous behavior of the area of the polar coronal hole and the strength of the polar magnetic field.

In this article, we describe the evolution of the $\mathrm{PCH}$ areas and polar magnetic-field strength from May 2010 until December 2013, approaching the maximum of Solar Cycle 24. While the polar magnetic field is changing sign, the PCHs areas have also become very small. One topic that we examine is whether the $\mathrm{PCH}$ areas can be used to establish the time of solar maximum. Two other topics that we discuss are whether the polarity reversal and $\mathrm{PCH}$ decreasing area process are asymmetric between the hemispheres.

In Section 2, we first describe the synoptic maps calculated from SDO/AIA and $\mathrm{SDO} / \mathrm{HMI}$ data, and then we explain the methodology used to measure $\mathrm{PCH}$ areas in detail. In Section 3, we present our results; in particular, our observations show that the PCH sizes decline at different times in the two hemispheres, suggesting that the Solar Cycle 24 polarity reversal process is asymmetric. In Section 4, we discuss these results in light of results from other research groups, and in Section 5 we present our summary and conclusions.

\section{Data Analysis}

We used synoptic maps created with AIA and HMI data during Solar Cycle 24. We measured the PCH areas from EUV Carrington Rotation (CR) synoptic maps, EUV Harvey Rotation 


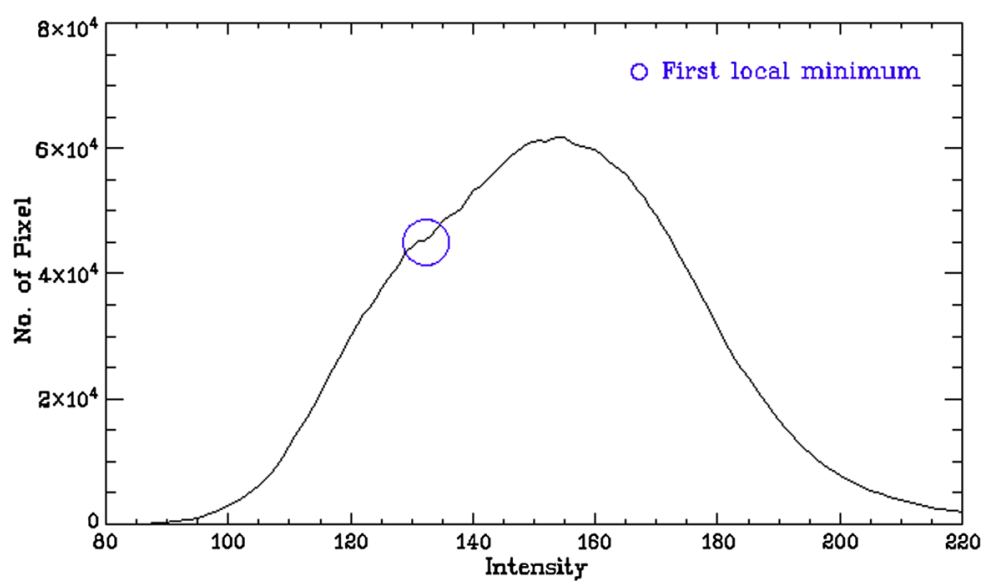

Figure 1 Histogram of pixel intensity obtained from the AIA 304 A Carrington Rotation synoptic maps. The circle represents the first local minimum of the data.

(HR) synoptic maps, and HMI radial magnetic-field synoptic maps. (A CR is the 27.27-day rotation period of a sunspot. An HR is the 33-day rotation period of the polar regions (Kirk et al., 2009).) We created the EUV synoptic maps from AIA $193 \AA$ (Fe XII, XXIV), AIA $171 \AA$ (Fe IX), and AIA $304 \AA$ (He II) data.

\subsection{AIA Data Preparation}

\subsubsection{AIA Carrington Rotation Synoptic Maps}

First, the solar images were mapped to the heliographic coordinate system and then to the heliocentric Cartesian coordinate system as seen from Earth (Thompson, 2006). The CR synoptic maps were constructed by concatenating longitudinal strips of $13.6^{\circ}$ centered at the central meridian, covering latitudes between $-90^{\circ}$ to $+90^{\circ}$. We used four images per day over a CR. The strips were mapped from left to right onto a rectangular grid. The size of the map is $3600 \times 1080$ pixels, with longitude and latitude in degrees along the $x$ - and $y$-axes, respectively. Pixels that correspond to latitudes that are out of view because of the tilt of the solar rotation axis with respect to the Ecliptic were set to "Not a Number" (NaN). The AIA CR maps are available at George Mason University (GMU) Space Weather Lab.

\subsubsection{AIA Harvey Rotation Synoptic Maps}

The HR synoptic maps concatenate longitudinal strips of $13.2^{\circ}$ centered at the central meridian, covering available latitudes between $-90^{\circ}$ to $+90^{\circ}$ after the coordinate transforms described above. Four images per day were combined over each HR. The size of these maps is $4096 \times 2048$ pixels with longitude along the $x$-axis and latitude along the $y$-axis, both in degrees.

\subsection{EUV PCH Area Calculation Method}

We used the technique described in Article I to measure the PCH areas in both EUV synoptic maps. Because coronal holes are regions of low intensities, we selected the first local minima 
of the histogram (the blue circled region shown in Figure 1) of each image as our initial threshold, then adjusted that initial value for the best visual fit of the $\mathrm{PCH}$ in the image. The number of pixels below the threshold at each latitude $[\theta]$ is recorded as $n(\theta)$. The fractional $\mathrm{PCH}$ area for the northern hemisphere is a sum over the latitudes satisfying $\theta>50^{\circ}$ :

$$
A_{\mathrm{PCH}}^{\mathrm{N}}=\frac{2 \pi^{2}}{p q} \sum_{\theta>50^{\circ}} n(\theta) \cos \theta,
$$

where $p$ is the total number of pixels in latitude and $q$ is the total number of pixels in longitude. The fractional $\mathrm{PCH}$ area for the southern hemisphere is a similar sum over the latitudes satisfying $\theta<-50^{\circ}$.

\subsection{HMI Data Preparation}

We used the HMI radial-field synoptic maps from Stanford University to study the evolution of the PCH area and Solar Cycle 24 pole reversal. These synoptic maps are constructed over a CR from the HMI 720-second line-of-sight (LOS) magnetograms described by Liu et al. (2012). Radial fields were produced by dividing the LOS magnetic field in each pixel by the cosine of the corresponding angle from disk center before remapping to heliographic coordinates. The maps are $3600 \times 1440$ pixels with longitude in degrees along the $x$-axis and latitude in degrees along the $y$-axis.

\subsection{Magnetic Field PCH Area Calculation Method}

The boundary of a $\mathrm{PCH}$ is the latitude where the dominant field changes sign (Article I). We averaged the magnetic-field strength in longitude at each latitude. The latitude where the averaged radial magnetic field changes sign from its value in the polar cap is $\theta_{b}$ and the area comes from the relationship $A_{B}=\left(1-\sin \left|\theta_{b}\right|\right) / 2$.

\section{Results}

A comparison of the PCH areas from HR and CR synoptic maps was used to determine whether the two synoptic maps gave equivalent results. When the CR and HR PCH areas are compared over time (Figure 2), we can see that the methods return similar results. For either hemisphere the areas from both EUV synoptic maps are interlaced, showing good agreement. From this comparison it appears that the noise level is $0.5 \%$, which may explain why they do not fall to zero near solar maximum. Neither rotation rate is preferred by this comparison, and we used the PCH areas measured from the CR synoptic maps for our further studies, to provide continuity with the data series of Article I.

Differences between the northern and southern PCH areas are clearly seen in Figure 2 during the rising phase of Solar Cycle 24. From May 2010 until mid-2012 the southern PCH area is about twice the northern PCH area. After that the southern PCH begins to slowly but steadily decrease in area. Neither PCH has completely disappeared in the EUV results.

Changes over the solar cycle can be seen in the EUV PCH areas shown in Figure 2. However, SDO was launched in February 2010 and began delivering data 1 May 2010. This was 18 months after the December 2008 solar minimum. It is necessary to prepend data from Article I to examine the period of solar minimum. The average of the areas from 


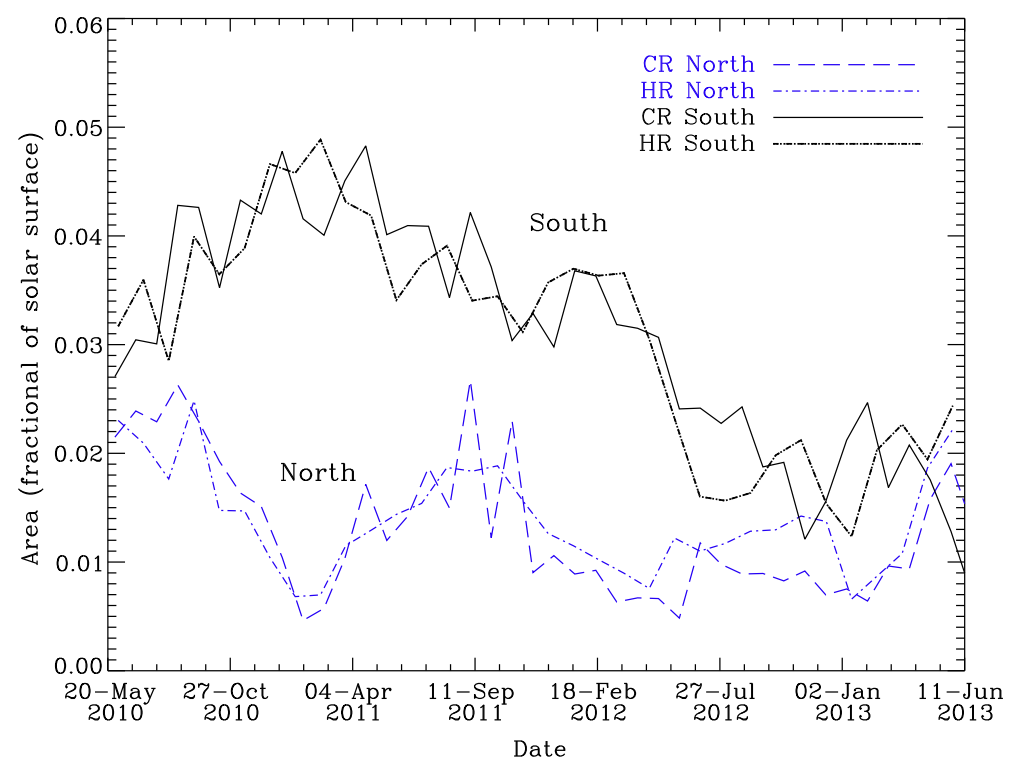

Figure 2 A comparison of the areas of the northern and southern PCHs obtained from the AIA $304 \AA$ Carrington Rotation and AIA $304 \AA$ Harvey Rotation (defined in Section 2: Data Analysis) synoptic maps. The black solid and black chain-dashed curves are the southern fractional PCH areas from CR and HR maps, respectively. The blue dashed and blue dash-dotted curves show the northern fractional $\mathrm{PCH}$ areas from the CR and HR synoptic maps, respectively.

all of the wavelengths in the current study and that of Hess Webber et al. (2014) and the sunspot number are displayed in Figure 3. The PCH areas and the sunspot numbers have an inverse correlation. As the sunspot number increases, the $\mathrm{PCH}$ area decreases, and vice versa. However, the flatness of $\mathrm{PCH}$ areas during maximum does not give a better indication of the timing of solar maximum than the sunspot number.

The PCH areas in the northern hemisphere were greatest in December 2006 (two years before solar minimum), indicated by blue arrow in Figure 3, and they have decreased as the solar cycle progressed towards solar maximum. The decline in the northern PCH area fluctuates around an overall declining trend until mid-2012. After late 2012, the northern PCH is very small (but did not completely disappear with the EUV method), and begins to increase in 2013. The area in the southern hemisphere peaked later than the northern hemisphere (represented by red arrow in Figure 3), but stayed at a higher value until the third quarter of 2011 and has since fallen to below the area of the northern hemisphere.

At this time, both the northern and southern $\mathrm{PCH}$ fractional areas were smaller than $0.5 \%$, meaning a bounding latitude of about $82^{\circ}$. This is near the effect of the solar obliquity and may represent a bound on the areas derived with this algorithm. We used the variation of the PCH area derived from the solar magnetic field to refine our estimates for the timing of solar maximum.

The top plot in Figure 4 shows the area of the PCHs estimated from the variation of the HMI radial magnetic field. Linear-trend fits were used to determine when this PCH area goes to zero. Areas that exceed $8 \%$ (corresponding to a polar-cap boundary at latitudes of $\pm 57^{\circ}$ ) were considered to be magnetic surges and ignored in the fits. Magnetic surges occur 


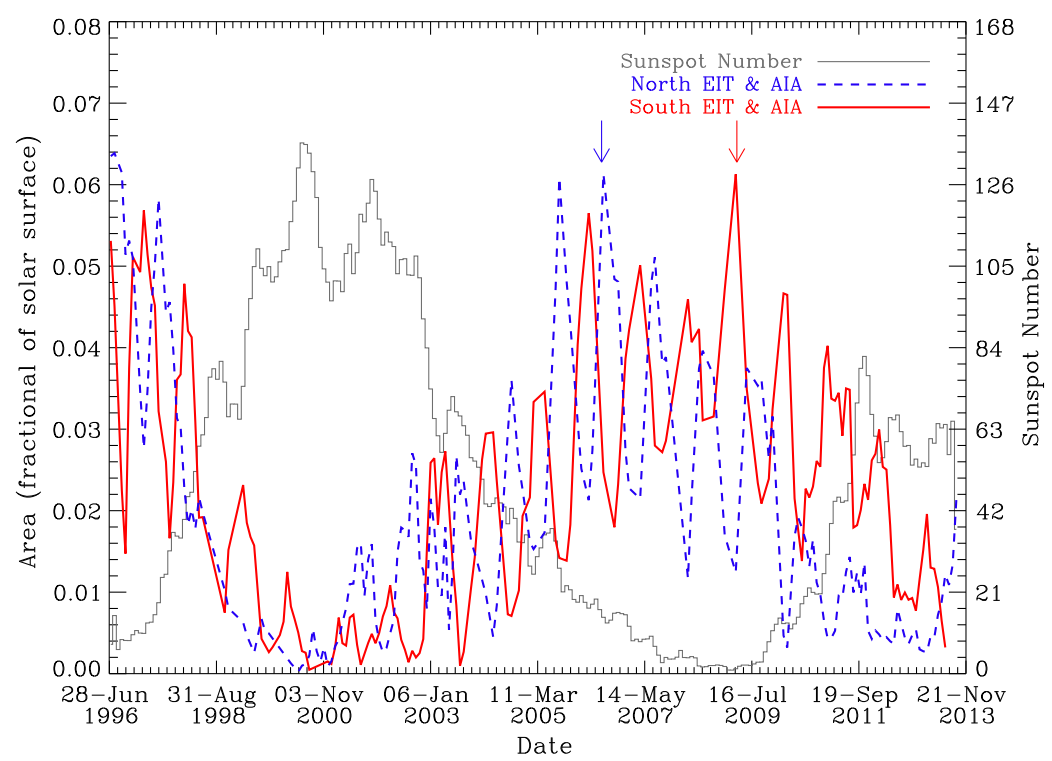

Figure 3 The fractional PCH areas are shown from 28 June 1996 to 28 September 2013 (CR 1911CR 2141). SOHO/EIT $304 \AA$ A, EIT $195 \AA$, and EIT $171 \AA$ data have been used from 28 June 1996 to 22 April 2010 (CR 1911-CR 2096) and SDO AIA $304 \AA$, AIA $193 \AA$, and AIA $171 \AA$ data have been used from 20 May 2010 to 28 September 2013 (CR 2097 - CR 2141). The blue dashed curve represents the average northern PCH area, the red solid curve represents the average southern PCH area from the three wavelengths. The blue and red arrow indicates the time when northern and southern $\mathrm{PCH}$ areas attained their maximum size. The thin gray solid curve represents the monthly sunspot number.

when the magnetic streamers that connect the lower latitude active bands to the polar regions bring magnetic flux into the polar cap.

These magnetic surges can be seen in the butterfly diagram constructed by placing the longitudinally averaged synoptic maps next to each other (Figure 5). The surges are the red and blue streamers running from active-region latitudes to the poles. We observe that the northern polar magnetic field has reversed while the southern polar field may have reversed, but the tilt of the rotation axis is not allowing the full pole to be observed. For most of 2013, both hemispheres had the same dominant magnetic polarity.

By extrapolating the linear fit to the synoptic magnetic-field data in Figure 4, the northern hemisphere may have reached solar maximum in mid-2012 and the southern hemisphere could have reached solar maximum in late 2013. However, another strong magnetic surge occurred at the southern hemisphere at the end of 2013. The bottom plot in Figure 4 shows the northern and southern hemisphere sunspot areas. A daily sunspot area dataset is available from NASA's Marshall Space Flight Center (Hathaway, 2010). The data show that the northern sunspot area reached a maximum in late 2011, which is 0.75 years before our northern magnetic data changed its polarity. The southern sunspot area may have peaked in late 2013 or early 2014.

Figure 6 shows the radial magnetic field averaged from $\pm 85^{\circ}$ to $\pm 89^{\circ}$ as a function of calendar date. We can see that the northern pole changed its polarity in mid-2012, although the southern magnetic field has yet to change its polarity (but it should change in the near future). These observations again imply that the two poles reached their solar-maximum conditions at separate times. 

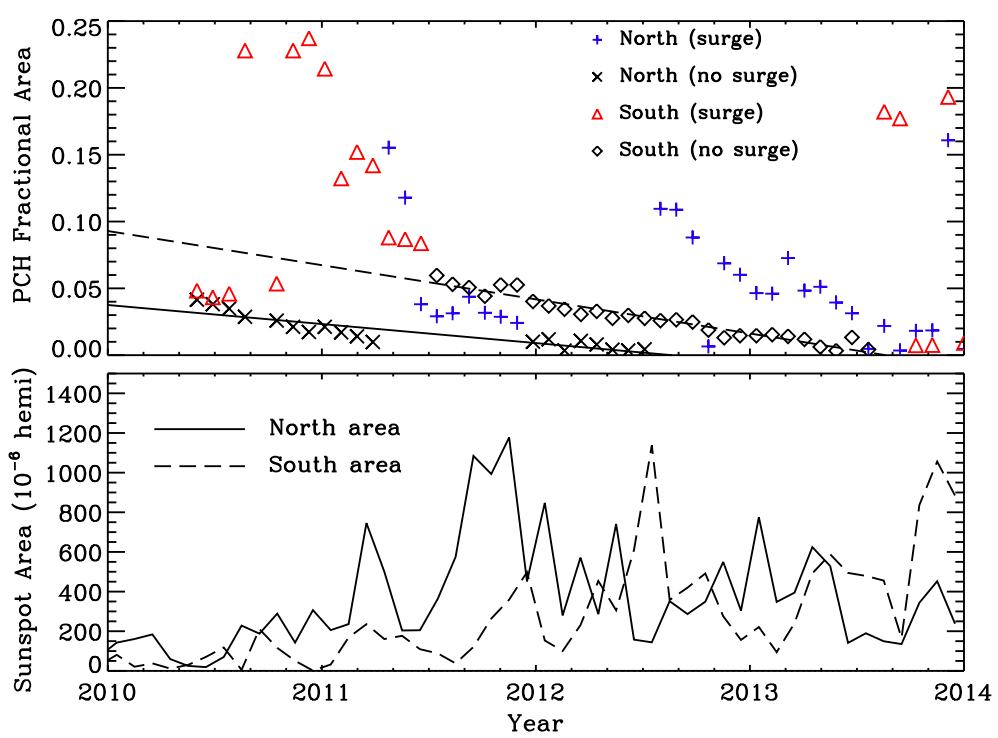

Figure 4 Top: The fractional area of the polar coronal hole estimated from the behavior of the HMI radial magnetic field. Bottom: The sunspot areas for each hemisphere (in units of millionths of the area of a solar hemisphere). Northern hemisphere PCH areas are shown as black crosses for normal data, while blue plus symbols indicate that a surge was in progress. Southern hemisphere $\mathrm{PCH}$ areas are shown as black diamonds for normal data and red triangles during a surge. A linear-trend fit was used to determine when the $\mathrm{PCH}$ area determined by the magnetic field goes to zero. Fit lines were calculated using only the normal points in each hemisphere. (PCH areas measured during surges were ignored in the fits.) The fit line for the northern hemisphere is a solid black line, a dashed line is drawn for the southern hemisphere. Solid and dashed curves in the bottom plot represent the northern and southern sunspot area.

\section{Discussion}

Our results show that the polar magnetic-field polarity reversals of the two hemispheres are out of phase during the Solar Cycle 24 maximum. The data show that the southern hemisphere will go through its polar magnetic-field reversal at least 1.5 years after the North. This is a much shorter time than the hemispheric shift at solar minimum. We concluded in Article I that the northern hemisphere reached solar-minimum conditions, as judged by the size of their PCHs, 3.25 years before the southern hemisphere during the minimum between Solar Cycles 23 and 24 (times shown by arrows in Figure 3).

Other research groups report similar hemispheric phase shifts: Altrock (2003) suggested that, prior to solar maximum, an emission feature in Fe XIV $5303 \AA$ appears above $50^{\circ}$ in both hemispheres and begins to move toward the poles: a concept he called the "Rush to the Poles". Altrock (2011) estimated that solar maximum occurs when a linear fit to the rush-tothe-pole data reaches $76^{\circ} \pm 2^{\circ}$. Altrock (2014) suggested that the northern hemisphere had already reached its maximum in mid-2011, while the southern hemisphere lagged behind and will reach solar maximum in early 2014. Gopalswamy et al. (2012) studied magnetic and microwave butterfly diagrams and prominence-eruption activity, and concluded that the rush-to-the-pole phenomena seemed to be complete in the northern hemisphere as of March, 2012.

Hoeksema (2012) used WSO data to show that the solar magnetic field in the North reversed in late 2012 while the southern high-latitude reversal lags behind. Zhao, Landi, and 


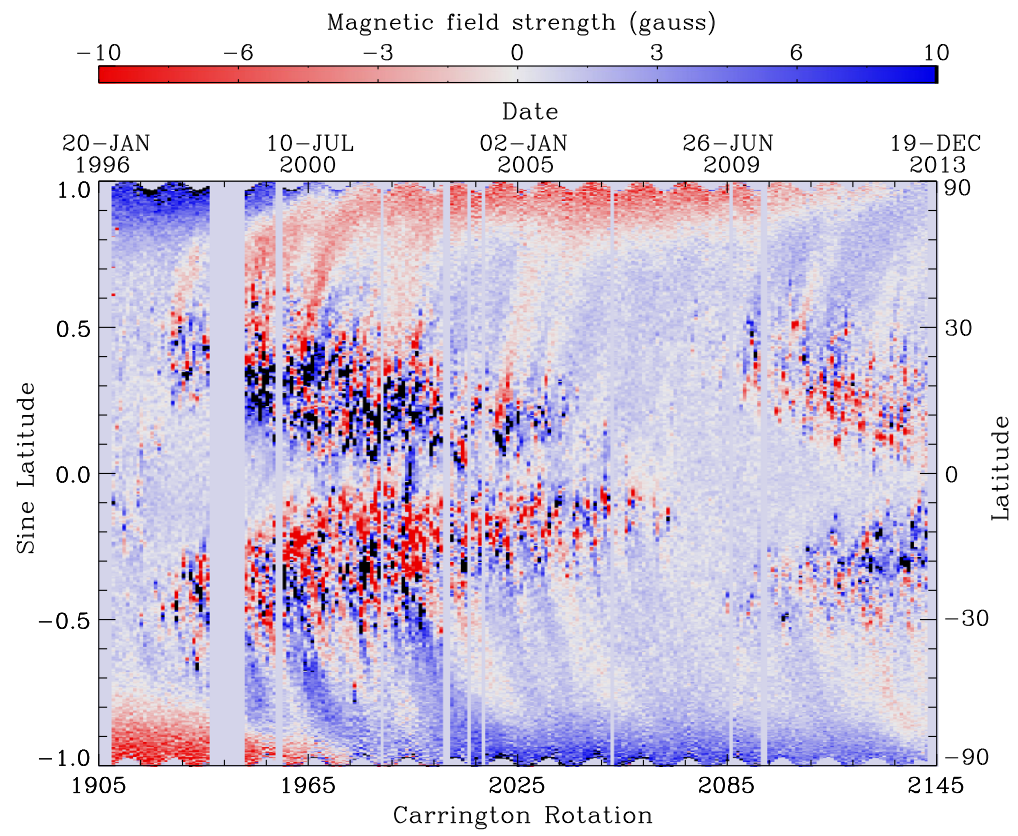

Figure 5 A butterfly diagram created with MDI and HMI data. SOHO/MDI data were used through the end of CR 2095 (22 April 2010), after which HMI data are used until CR 2141 (31 August 2013). Blue regions have positive polarity and red regions have negative polarity. The blue and red streamers are the magnetic surges that connect the polar regions to the active latitudes. The sinusoidal signatures at the poles are due to the $B_{0}$-angle that shifts the poles into and out of sight. This image has a cutoff field magnitude of \pm 10 Gauss.

Figure 6 Averaged polar magnetic field as a function of calendar date. The blue chain-dashed curve is the magnetic field averaged from $85^{\circ}$ to $89^{\circ}$ in the northern hemisphere. The solid black curve is the magnetic-field averaged data from $-85^{\circ}$ to $-89^{\circ}$ in the southern hemisphere, and the sign has been reversed for comparison purposes. The black dashed line is the difference between the northern and southern magnetic-field strengths.

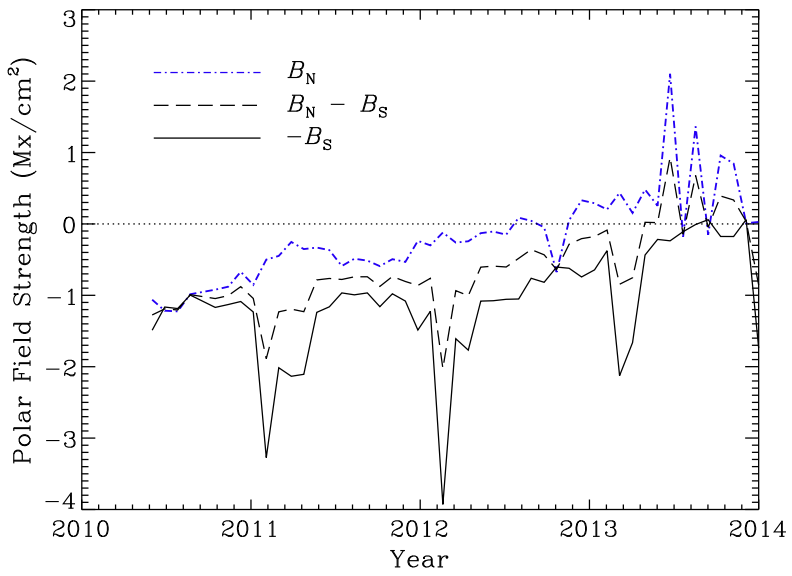

Gibson (2013) obtained the standard deviation and the integrated slope of the latitude of the heliospheric current sheet from the magnetic synoptic maps calculated by the potential-field source-surface (PFSS) model. These parameters were larger in the northern hemisphere than in the southern hemisphere, indicating that the northern polar field was ready for its polarity reversal sooner than the South. Svalgaard and Kamide (2013) combined sunspot number, the behavior of magnetic-field observations, green-line coronal emissions, and the behavior 
of polar coronal holes to examine the asymmetries of the North-South Pole reversal to conclude that the northern hemisphere reversed its polarity first.

Our results using both AIA and HMI data are consistent with these others in showing that the polarity reversal of the two hemispheres is out of phase. We estimate that this difference is at least 1.5 years during the maximum phase of Solar Cycle 24. While the northern hemisphere polar field has already reversed, the southern hemisphere polar field may not have reversed. A strong surge in the last six months of 2013 may lead to conditions in the South that resemble those in the North during early 2011. A strong magnetic surge at that time enlarged the northern PCH area and delayed its disappearance until mid-2012.

\section{Summary and Conclusion}

We have calculated the areas of the polar coronal holes in both hemispheres of the Sun during Solar Cycle 24 using SDO data. Three different methods were used to calculate the $\mathrm{PCH}$ areas from synoptic maps generated with AIA and HMI data. Two were similar to those described in Article I while the third used Harvey Rotation synoptic maps from mid-2010 through mid-2013 to calculate the area. Our analysis shows the following:

- The PCH fractional areas calculated from the CR synoptic maps and HR synoptic maps have similar values and variations with time. This also demonstrated that the systematic variations in the EIT data described in Article I are greatly reduced in amplitude in the AIA data.

- Both the northern and southern PCH areas have decreased significantly since the launch of SDO in 2010. The northern PCH area began to decrease earlier and has reached conditions of solar maximum. The southern PCH has decreased in size, but a recent magnetic surge may cause it to increase again. In neither hemisphere has the $\mathrm{PCH}$ completely disappeared in the EUV results, but the PCH areas determined from the magnetic field have fallen to zero.

- Both poles were being refreshed by magnetic surges with the dominant polarity of Solar Cycle 24. Between magnetic surges the $\mathrm{PCH}$ areas derived from the magnetic field have a simple linear dependence with time (see Figure 4). Linear fits to these $\mathrm{PCH}$ areas indicate that the northern hemisphere had already gone through its polar-field reversal and reached solar-maximum conditions in mid-2012, while the southern hemisphere may reach solarmaximum conditions as late as 2014 . These linear fits again show that the solar maxima of the hemispheres will be offset in time.

Our analysis shows that the $\mathrm{PCH}$ areas were quite small at the time of magnetic-field reversal and at the time of high sunspot number. The $\mathrm{PCH}$ area can be used to establish the time of solar maximum. However, it is evident in Figure 3 that the areas do not give a better indication of the timing of solar maximum than does the sunspot number.

The phasing of solar maximum continues to be a fascinating topic. The largest monthly sunspot number so far in Solar Cycle 24 was 97 in November 2011. The sunspot area in the northern hemisphere also reached a local maximum in that month. Our analysis also shows that the northern PCH reached its lowest value, and the magnetic field reversed 0.75 years later. Although the southern PCH is now quite small and the field reversal appears imminent, the sunspot number is large at the same time, without a delay between the onset of the peak sunspot number and the polar conditions that indicate solar maximum. Merging these asymmetric phase differences into a coherent model is a challenge that could result in better predictions of solar activity. 
Acknowledgements N. Karna thanks the Schlumberger Foundation Faculty for the Future for supporting this research. S.A. Hess Webber thanks the Catholic University of America and NASA's Solar Dynamics Observatory for supporting this research. The AIA and HMI data are courtesy of NASA/SDO and the AIA and HMI Science Investigation Teams. The HMI radial synoptic maps are available at the Stanford website. The GMU AIA Synoptic Maps Dataset can be accessed at the George Mason University (GMU) Space Weather Lab. The EIT images are courtesy of the SOHO/EIT consortium. SOHO is a mission of international cooperation between ESA and NASA. The MDI images are provided by the Solar Oscillations Investigation (SOI) team at the Stanford-Lockheed Institute for Space Research. Daily sunspot areas dataset were obtained from NASA/Marshall Solar Physics. Daily values of International Sunspot Number, $\left[R_{Z}\right]$, were obtained from the National Geophysical Data Center in Boulder, CO, USA.

\section{References}

Altrock, R.C.: 2003, Use of ground-based coronal data to predict the date of solar-cycle maximum. Solar Phys. 216, $343-352$. DOI.

Altrock, R.C.: 2011, Coronal Fe XIV emission during the Whole Heliosphere Interval Campaign. Solar Phys. 274, $251-257$. DOI.

Altrock, R.C.: 2014, Forecasting the maximum of Solar Cycle 24 with coronal Fe XIV emission. Solar Phys. 289, 623-629. DOI.

Babcock, H.D.: 1959, The Sun's polar magnetic field. Astrophys. J. 130, $364-365$. DOI.

Bilenko, I.A.: 2002, Coronal holes and the solar polar field reversal. Astron. Astrophys. 396, 657-666. DOI.

Bohlin, J.D.: 1977, Extreme-ultraviolet observations of coronal holes. I - Locations, sizes and evolution of coronal holes, June 1973 - January 1974. Solar Phys. 51, 377-398. DOI.

Bohlin, J.D., Sheeley, N.R. Jr.: 1978, Extreme ultraviolet observations of coronal holes. II - Association of holes with solar magnetic fields and a model for their formation during the solar cycle. Solar Phys. 56, 125 - 151. DOI.

Cranmer, S.R.: 2009, Coronal holes. Living Rev. Solar Phys. 6, 3. DOI.

DeRosa, M.L., Brun, A.S., Hoeksema, J.T.: 2012, Solar magnetic field reversals and the role of dynamo families. Astrophys. J. 757, 96. DOI.

Gopalswamy, N., Yashiro, S., Mäkelä, P., Michalek, G., Shibasaki, K., Hathaway, D.H.: 2012, Behavior of solar cycles 23 and 24 revealed by microwave observations. Astrophys. J. Lett. 750, L42. DOI.

Hathaway, D.H.: 2010, The solar cycle. Living Rev. Solar Phys. 7, 1. (Accessed 13-Jun-2013.) DOI.

Hess Webber, S.A., Karna, N., Pesnell, W.D., Kirk, M.S.: 2014, Areas of polar coronal holes from 1996 through 2010. Solar Phys., submitted.

Hoeksema, J.T.: 2012, Polar reversal, solar maximum, and the large-scale heliospheric field in Solar Cycle 24. Bull. Am. Astron. Soc. 220, 206.07.

Kirk, M.S., Pesnell, W.D., Young, C.A., Hess Webber, S.A.: 2009, Automated detection of EUV polar coronal holes during Solar Cycle 23. Solar Phys. 257, 99 - 112. DOI.

Liu, Y., Hoeksema, J.T., Scherrer, P.H., Schou, J., Couvidat, S., Bush, R.I., Duvall, T.L., Hayashi, K., Sun, X., Zhao, X.: 2012, Comparison of line-of-sight magnetograms taken by the solar dynamics observatory/helioseismic and magnetic imager and solar and heliospheric observatory/Michelson Doppler imager. Solar Phys. 279, 295 -316. DOI.

Sawtell Cameron, W.: 1957, A unique high-latitude sunspot group. Publ. Astron. Soc. Pac. 69, $561-563$. DOI.

Scherrer, P.H., Wilcox, J.M., Svalgaard, L., Duvall, T.L. Jr., Dittmer, P.H., Gustafson, E.K.: 1977, The mean magnetic field of the Sun - observations at Stanford. Solar Phys. 54, 353-361. DOI.

Svalgaard, L., Kamide, Y.: 2013, Asymmetric solar polar field reversals. Astrophys. J. 763, 23. DOI.

Thompson, W.T.: 2006, Coordinate systems for solar image data. Astron. Astrophys. 449, 791 - 803. DOI.

Waldmeier, M.: 1981, Cyclic variations of the polar coronal hole. Solar Phys. 70, 251 - 258. DOI.

Webb, D.F., Davis, J.M., McIntosh, P.S.: 1984, Observations of the reappearance of polar coronal holes and the reversal of the polar magnetic field. Solar Phys. 92, 109. DOI.

Zhao, L., Landi, E., Gibson, S.E.: 2013, Two novel parameters to evaluate the global complexity of the Sun's magnetic field and track the solar cycle. Astrophys. J. 773, 157. DOI. 